\title{
TOWARDS UNDERSTANDING SPONTANEOUS SPEECH: WORD ACCURACY VS. CONCEPT ACCURACY
}

\author{
M. Boros ${ }^{1}$, W. Eckert ${ }^{2}$, F. Gallwit $z^{2}$, \\ G. Görz ${ }^{1}$, G. Hanrieder ${ }^{1}$, H. Niemann ${ }^{1,2}$ \\ ${ }^{1}$ Bayerisches Forschungszentrum für Wissensbasierte Systeme (FORWISS), \\ Am Weichselgarten 7, D-91058 Erlangen, Germany \\ ${ }^{2}$ Lehrstuhl für Mustererkennung (Informatik 5),Universität Erlangen-Nürnberg, \\ Martensstraße 3, D-91058 Erlangen, Germany \\ email: boros@forwiss . uni-erlangen.de
}

\begin{abstract}
In this paper we describe an approach to automatic evaluation of both the speech recognition and understanding capabilities of a spoken dialogue system for train time table information. We use word accuracy for recognition and concept accuracy for understanding performance judgement. Both measures are calculated by comparing these modules' output with a correct reference answer. We report evaluation results for a spontaneous speech corpus with about 10000 utterances. We observed a nearly linear relationship between word accuracy and concept accuracy.
\end{abstract}

\section{INTRODUCTION}

Total system evaluation plays an important role for developers of spoken dialogue systems, because it allows both to monitor progress within a single project and to compare different solutions for the same task. An objective and verifiable judgement of system performance requires that the scientific community agrees upon widely accepted evaluation measures. In speech recognition, such a mutually agreed upon measure is available with the so-called word accuracy (WA). There exist standardized tools which can automatically compute the WA of recognition results for a given test corpus annotated with transcriptions of the actually spoken words. This high standard of automatic evaluation methods could not yet be transferred to the higher processing level of speech understanding, although the last few years have witnessed increasing efforts in the development of an evaluation methodology for natural language processing (cf. [6], [11], [2]).

This paper describes our approach to automatic evaluation of both the recognition and the understanding capabilities of a spoken dialogue system for train time table inquiries [3]. Such an integrated evaluation environment allows a systematic investigation of the relationship between recognition and understanding performance. The central question is: How does a change in the recognition accuracy affect the understanding accuracy? First we describe the evaluation measures word accuracy and concept accuracy. After this we show our evaluation architecture for automatic calculation of re- cognition and understanding accuracy. Finally, we report results for a spontaneous speech corpus containing about 10000 utterances.

\section{EVALUATION MEASURES}

Automatic evaluation methods require the use of prepared test corpora in which each test case is combined with a "correct" reference answer against which the system output can be judged. In speech recognition, it is relatively uncontroversial how this reference answers look like: they are transcriptions of the words that were actually spoken. ${ }^{1}$ It is less clear, however, what constitutes the "correct" analysis at the level of language understanding. Currently, there is no agreement among computational linguists regarding a "correct" semantic representation for a wide variety of linguistic phenomena. As a consequence, there are no semantically annotated corpora available as a common test bed for comparative evaluation of linguistic processing components. Nevertheless, we believe that an objective and verifiable measurement of the understanding capabilities of a system can only be achieved with a "reference answer"-based approach using test corpora with semantic annotations. This conviction is based on the fact that the main task of the linguistic processing component in a spoken dialogue system is to map the spoken input to a semantic representation. Evaluation approaches which look only at the surface forms ${ }^{2}$ or the syntactic structures [1] of the parsing results cannot judge the parser performance regarding the construction of a semantic representation. Therefore, we defined a semantic annotation format within our task domain. For measuring the understanding performance we adopted the so-called concept accuracy. This measure, which was proposed from the evaluation working group of the ESPRIT project SUNDIAL [10], can be calculated automatically in analogy with the recognition measure word accuracy.

\footnotetext{
${ }^{1}$ There are still debates on the transcription and evaluation of spontaneous speech containing fragmentary words, hesitations, background noise, etc.

${ }^{2}$ In [9] a word graph parser is rated by calculating the sentence recognition accuracy, which is defined as "the number of word graphs where the analysis found the spoken sentences divided by the number of word graphs".
} 


\subsection{Word Accuracy}

Word Accuracy (WA) is a widely accepted evaluation measure for word recognizers. The automatic calculation of WA for a given set of recognition results requires the existence of reference transliterations for all spoken utterances. The reference answers consist of a transcription of what was actually spoken. Given the reference REF, the WA of the recognizer output HYP is determined by calculating the Levenshtein distance between REF and HYP and by assigning equal costs to substitution, insertion, and deletion errors. WA is calculated as a percentage using the formula

$$
W A=100\left(1-\frac{W_{S}+W_{I}+W_{D}}{W}\right) \%
$$

where $W$ is the total number of words in REF, and $W_{S}, W_{I}$, $W_{D}$ are the number of reference words which were substituted, inserted, and deleted in HYP, respectively.

For example, the WA of the recognized string in (2) is $66.7 \%$, since the spoken word I was deleted and the spoken word Berlin was substituted by Bonn in HYP, such that $W_{D}=1$ and $W_{S}=1$. By inserting these values into formula (1) the WA is calculated by $100\left(1-\frac{2}{6}\right)=66.7 \%$.

\begin{tabular}{|l|lll|}
\hline REF: & I & want to go to & Berlin \\
HYP: & & want to go to & Bonn \\
\hline
\end{tabular}

\subsection{Concept Accuracy}

While WA evaluates the performance of the speech recognition component, the language understanding capabilities of a system can be judged by concept accuracy (CA). ${ }^{3}$ This approach is based on the assumption that the main task of the linguistic processor in a spoken dialogue system is to extract the propositional content from the spoken utterance. Furthermore, it is assumed that this propositional content can be represented as a list of semantic units ( $S U$ ) taking the form of attribute-value pairs. The definition of the attributes relevant for understanding is determined by domaindependent task parameters which reflect the functionality of the system. For example, in a train time table information task the system cannot access the connected database system without knowing the values for the task parameters sourcecity, goalcity and date. Accordingly, the propositional content of a sentence like (3) is represented as the series of SUs shown in (4).

(3) I want to go from Bonn to Berlin.

(4) [sourcecity:Bonn, goalcity:Berlin]

Given such semantic reference answers in form of task parameter-value pairs the performance of a speech understanding component can be measured in analogy with the

\footnotetext{
${ }^{3}$ In [10] a similar measure was called information content.
}

method used for word recognition evaluation. Concept accuracy $\mathrm{CA}$ can be calculated by replacing the words $W$ in formula (1) with semantic units $S U$ :

$$
C A=100\left(1-\frac{S U_{S}+S U_{I}+S U_{D}}{S U}\right) \%
$$

$S U$ is the total number of semantic units in the reference answer and $S U_{S}, S U_{I}$, and $S U_{D}$ are the number of semantic units that were substituted, inserted, and deleted in the parser output, respectively. The calculation of $\mathrm{CA}$ will be illustrated in the following example:

\begin{tabular}{|l|ll|}
\hline Spoken: & No & to Bon $n$ \\
REF: & dm_marker $:$ no & goalcity $:$ Bonn \\
\hline Recog.: & No & to Berlin \\
HYP: & dm_marker:no & goalcity $:$ Berlin \\
\hline
\end{tabular}

The total number of uttered semantic units in (6) is $S U=2$. Due to the misrecognition of the spoken word Bonn the correct semantic unit goalcity:Bonn was replaced by goalcity:Berlin in the parser output, thus being $S U_{S}=1$. This yields a concept accuracy of $50 \%$ by calculating $C A=$ $100\left(1-\frac{1}{2}\right) \%=50 \%$.

The example shows that beside its ability to judge the parser performance on a semantic level, $\mathrm{CA}$ is also an adequate measure for evaluating robust parsers which allow partial analysis. This is a distinguishing feature of CA in comparison with binary measures like sentence recognition accuracy. In such approaches a system output either totally agrees with a reference answer or it is counted as a total failure. Concept accuracy on the other hand is able to measure the degree of system understanding. In the above example, $50 \% \mathrm{CA}$ expresses the fact that the chain comprising word recognizer and parser was able to extract half of the propositional content from the input utterance.

\subsection{Word Accuracy vs. Concept Accu- racy}

The example shown in the previous section illustrates that the relationship between WA and CA cannot be predicted systematically. Both measures can differ considerably because WA does not make a difference between filler words and semantically relevant words. For example, WA in (6) is $75 \%$ (only 1 substitution error), whereas CA is only $50 \%$. This is explained by the fact that the substituted city name forms the semantic core of the goalcity-concept which is misunderstood as a whole in consequence. The opposite case is illustrated by example (7) where $W A=66.7 \%$ but $C A=100 \%$ because the misrecognitions did not concern the parts relevant for understanding.

\begin{tabular}{|l|ll|}
\hline $\begin{array}{l}\text { Spoken: } \\
\text { REF: }\end{array}$ & I want to go & $\begin{array}{l}\text { to Berlin } \\
\text { goalcity:Berlin }\end{array}$ \\
\hline $\begin{array}{l}\text { Recog:: } \\
\text { HYP: }\end{array}$ & I wonder go & $\begin{array}{l}\text { to Berlin } \\
\text { goalcity:Berlin }\end{array}$ \\
\hline
\end{tabular}


The example shows that it is possible to achieve perfect utterance understanding with less than perfect word recognition. This happens when misrecognitions only affect semantically irrelevant (in our domain) filler words. On the other hand, if recognition errors occur within parts that are relevant for understanding an utterance, CA may become lower than WA. This relationship between WA and CA was investigated in the experiments we describe in section 4 . These experiments were performed with the evaluation environment and the data described in the next section.

\section{EVALUATION ENVIRONMENT}

We implemented a test environment which can automatically calculate the concept accuracy of the parsing results for a given semantically annotated test corpus. The architecture of our automatic evaluation system is outlined in Figure 1.

The test corpus consists of a set of test cases, which are either transliterations of the spoken utterance or word recognition results. In the first case the environment is used for evaluating the linguistic component alone, in the latter case word recognizer (FEP) and linguistic processing component (LP) are evaluated together. In both cases each test sentence is combined with a semantic reference annotation in the form of attribute-value pairs shown above. The test cases are handed over sequentially to the parser which tries to analyze it with respect to its knowledge base, i.e. the grammar. At the moment we use a robust chart parser [8] which selects a set of partial results from the chart if no complete analysis can be found. This parser uses a highly lexicalized unification grammar based on the UCG formalism [12]. The strict modularity of the evaluation environment allows an easy replacement of test data as well as of the linguistic processing component. Thus, although we use the evaluation programme mainly for progress evaluation, it can also be used for comparative evaluation of alternative implementations of the lingusitic component. The only requirement is that the components generate comparable results in the semantic interface language (SIL, [7]) used in our dialogue system. In order to compare these complex parsing result structures with the much simpler reference annotations, we implemented a (domain specific) module sil2ref which maps between SIL and the annotated semantic units. Finally, the parsing results and the semantic annotations are compared by calculating the Levenshtein distance by programme eval_seg. The resulting concept accuracy is reported (cf. Figure 1).

\section{EXPERIMENTS \& RESULTS}

In our evaluation experiments we wanted to examine the relations between WA and CA, in order to see if improvement of the word recognizer (and thus WA) also leads to improvement of concept accuracy. Therefore several evaluation tests were run. Based on the same speech material we run the recognizer with different parameter settings, resulting in differences in word accuracy (and processing speed). These word chains have been processed by the linguistic processor

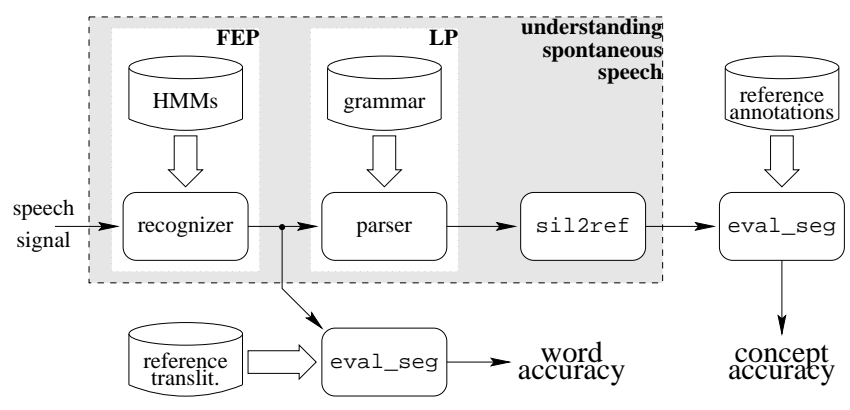

Figure 1: Architecture of the automatic evaluation system.

and corresponding figures for WA and CA were calculated.

Evaluation was performed on a test corpus collected while the system was accessible via the public telephone network [4]. 1092 dialogues with (naive) users were recorded. We recorded the word recognizer output, the transliterations and the semantic annotation for each utterance were done manually. Table 1 gives an overview of the test corpus.

\begin{tabular}{|l|r|}
\hline Total number of dialogues & 1092 \\
\hline Total number of utterances & 10114 \\
\hline Total number of words & 33477 \\
\hline Total number of semantic units & 14584 \\
\hline Different classes of semantic units & 38 \\
\hline
\end{tabular}

Table 1: Figures of the test corpus.

The first step was to evaluate the linguistic component of the system on its own, in order to measure the (semantic) coverage of the grammar. The resulting figure for CA reflects the grammars ability to extract the meaning of an utterance and thus its adequacy for the given domain. For this purpose, CA was computed using the transliterations as input to the parser and comparing the resulting semantic representation with the reference annotation. We achieved a linguistic coverage of $92.8 \%$ for spontaneous speech.

In order to examine the influence of different recognizer parameters on the systems concept accuracy, several experiments were carried out. The recognizer parameter to be altered was the beam width. For each parameter setting the recognizer was run on the recorded 10114 utterances of the corpus. Concept accuracy was then measured using the resulting recognizer output as input to the parser. Table 2 shows the resulting marks for WA and corresponding CA.

\begin{tabular}{|l|l|l|l|l|l|l|}
\hline WA & 48.8 & 65.7 & 72.9 & 77.5 & 83.0 & 84.9 \\
\hline CA & 46.7 & 61.9 & 68.2 & 73.0 & 78.5 & 79.8 \\
\hline
\end{tabular}

Table 2: Resulting marks for WA and CA when altering the recognizer beam width.

Table 2 shows that the marks for WA and CA correspond closely. This means that in our case the misrecognition in the acoustic front end processor affects content words and 


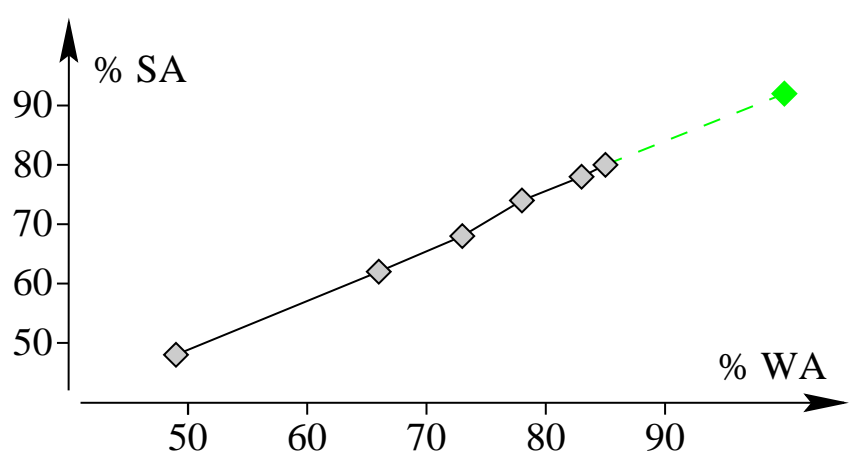

Figure 2: Relationship between word accuracy and concept accuracy.

filler words by the same amount. Moreover, we can see that the linguistic processor does not suffer from misrecognition of a few words. The parser has to be judged as extremely robust against recognition errors as well as phenomena of spontaneous speech. Figure 2 shows the nearly linear relation between word accuracy and corresponding concept accuracy.

In our case we can make the assumption that word accuracy is a suitable indicator for concept accuracy in a spoken dialogue system: recognizer and parser are well matched for their tasks and cooperate smoothly.

\section{SUMMARY}

In this paper we have shown an approach for the automated evaluation of an understanding module for spontaneous speech. This module consists of an acoustic recognizer and a linguistic processor. The resulting semantic content of each utterance is compared automatically with reference annotations, mimicking the evaluation of a word recognizer alone. Accordingly, the measure for a speech understanding system is called concept accuracy.

With our evaluation setup we are able to document improvements in one of our modules in an automated way. Thus, we are not only able to optimize isolated modules, but the whole understanding system. Experiments show that our parser is robust in the sense that we observe a nearly linear relation between WA and CA.

Further work will be commited to adjust parser parameters. Eventually we hope to increase CA beyond WA.

\section{ACKNOWLEDGEMENTS}

Part of this work was carried out in the project SYSLID which is funded by the Daimler-Benz research institute in Ulm. Part of this work was supported by the German Research Foundation (DFG) under contract number 810 830-0.

\section{REFERENCES}

1. E. Black, S. Abney, D. Flickenger, C. Gdaniec, R. Grishman, P. Harrison, D. Hindle, R. Ingria, F. Jelinek, J. Klavans, M. Liberman, M. Marcus, S. Roukos, B. Santorini, and T. Strzalkowski. A procedure for quantitatively comparing the syntactic coverage of english grammars. In Proceedings of DARPA Speech and Natural Language Workshop, pages 306-311, Pacific Grove, 1991.

2. R. Crouch, R. Gaizauskas, and K. Netter. Report of the study group on assessment and evaluation. Technical report, SRI International, Cambridge, 1996.

3. W. Eckert, Th. Kuhn, H. Niemann, S. Rieck, A. Scheuer, and E.-G. Schukat-Talamazzini. A spoken dialogue system for german intercity train timetable inquiries. In Proceedings of EUROSPEECH 93, volume 3, pages 1871-1874, Berlin, 1993.

4. W. Eckert, E. Nöth, H. Niemann, and E.-G. SchukatTalamazzini. Real users behave weird - experiences made collecting large human-machine-dialog corpora. In Proceedings of the ESCA Tutorial and Research Workshop on Spoken Dialogue Systems, pages 193-196, Vigsø, Denmark, 1995.

5. Bernd Hildebrand. Struktur und Bedeutung temporaler Konstituenten in einem sprachverstehenden Dialogsystem. PhD thesis, Universität Bielefeld, 1995.

6. L. Hirschman and H. Thompson. Overview of evaluation in speech and natural language processing. In R. Cole, editor, Survey of the State of the Art in Human Language Technology. Cambridge University Press, Cambridge, 1996. to appear.

7. S. McGlashan, F. Andry, and G. Niedermair. A proposal for SIL. Technical report, ESPRIT Project P2218 SUNDIAL, 1990.

8. K. Mecklenburg, P. Heisterkamp, and G. Hanrieder. A robust parser for continuous spoken language using Prolog. In Proceedings of the Fifth International Workshop on Natural Language Understanding and Logic Programming (NLULP 95), pages 127-141, Lisbon, Portugal, 1995.

9. L. Schmid. Parsing word graphs using a linguistic grammar and a statistical language model. In Proceedings of ICASSP-94, volume II, pages 41-44, 1994.

10. A. Simpson and N. Fraser. Black box and glass box evaluation of the SUNDIAL system. In Proceedings of EUROSPEECH 93, volume 2, pages 1423-1426, Berlin, 1993.

11. EAGLES Evaluation Subgroup. Evaluation of natural language processing systems. Technical Report EAGEWG-PR.2, Centre for Language Technology, Copenhagen, Denmark, 1994.

12. H. Zeevat. Combining categorial grammar and unification. In U. Reyle and Ch. Rohrer, editors, Natural Language Parsing and Linguistic Theories, pages 202-229. Reidel, Dordrecht, 1988. 